\title{
Les cultures hydroponiques aux Émirats arabes unis
}

Un nouvel entrepreneuriat entre insertion locale et réseaux globaux

Hydroponics in the United Arab Emirates: entrepreneurs as new players embedded in local and global networks

\section{Damien Calais}

\section{OpenEdition}

\section{Journals}

\section{Édition électronique}

URL : http://journals.openedition.org/artefact/5702

DOI : 10.4000/artefact.5702

ISSN : 2606-9245

\section{Éditeur :}

Association Artefact. Techniques histoire et sciences humaines, Presses universitaires du Midi

\section{Édition imprimée}

Date de publication : 15 juillet 2020

Pagination : 177-202

ISBN : 978-2-8107-0691-4

ISSN : 2273-0753

\section{Référence électronique}

Damien Calais, «Les cultures hydroponiques aux Émirats arabes unis », Artefact [En ligne], 12 | 2020, mis en ligne le 21 décembre 2020, consulté le 23 décembre 2020. URL : http://

journals.openedition.org/artefact/5702 ; DOI : https://doi.org/10.4000/artefact.5702

Artefact, Techniques, histoire et sciences humaines est mise à disposition selon les termes de la Licence Creative Commons Attribution - Pas d'Utilisation Commerciale - Pas de Modification 4.0 International. 


\title{
Les cultures hydroponiques aux Émirats arabes unis
}

\author{
Un nouvel entrepreneuriat entre insertion locale \\ et réseaux globaux
}

Damien Calais

\section{Résumé}

L'hydroponie est une méthode de culture irriguée hors sol réputée économe en eau. Les dirigeants politiques des Émirats arabes unis en font une priorité pour développer l'agriculture dans ce pays, très dépendant des importations pour son approvisionnement alimentaire, tout en ménageant ses ressources en eau qui se dégradent. Les entrepreneurs qui adoptent cette méthode apparaissent comme de nouveaux acteurs de la production agricole aux Émirats. L'hypothèse initiale de l'article est que cette innovation technique constitue un tournant dans l'histoire de ce système productif, une hypothèse relativisée ensuite au regard des obstacles à sa diffusion. Les entrepreneurs n'auraient pas eu recours à ces techniques coûteuses sans les réseaux familiaux, tribaux et nationaux par lesquels ils bénéficient de la redistribution de la rente pétrolière. Il est également nécessaire de prendre en compte leur insertion dans les réseaux globaux qui leur permettent d'accéder aux techniques de pointe requises pour ce type de cultures.

\section{Mots-clés}

champ organisationnel, développement agricole, développement des milieux désertiques, irrigation, mondialisation, système alimentaire, techniques de culture

99 Damien Calais, « Les cultures hydroponiques aux Émirats arabes unis. Un nouvel entrepreneuriat entre insertion locale et réseaux globaux », Artefact, 12, 2020, p. 177-202. 


\section{Hydroponics in the United Arab Emirates: entrepreneurs as new players embedded in local and global networks}

\section{Abstract}

Soilless culture with hydroponic irrigation techniques is said to be water-wise. The United Arab Emirates' rulers are making it a priority to develop agriculture in this heavily food import dependent country while mitigating the increasing water scarcity and degradation. The entrepreneurs who choose this farming method come up as new players in the country's agricultural sector. The working hypothesis of the article is that the introduction of such a technical innovation is a turning point in the history of the Emirati agricultural productive system. Yet this statement must be qualified in the light of the impediments in the expansion of hydroponics. The implementation of these costly techniques would not have taken place without the familial, tribal and national networks, which enable the entrepreneurs to have their share in the distribution of the oil rent. Besides this entrepreneurship is embedded in global networks that provide access to high technology essential for this method of cultivation.

\section{Keywords}

organizational field, agricultural development, desert development, irrigation, globalization, food system, farming techniques 


\section{Introduction}

Les cultures hydroponiques sont des cultures végétales hors sol dont les racines trempent dans une solution nutritive ou sont plongées dans un substrat irrigué par celle-ci. Ces cultures font l'objet de nombreuses publications dans le domaine des sciences de la vie mais sont rarement traitées par les recherches en sciences humaines et sociales. Dans le cadre d'une réflexion en histoire sociale des techniques, cet article mêle une enquête de terrain, un recoupement d'informations préexistantes et un travail d'archives. Il met en lumière le développement des cultures hydroponiques aux Émirats arabes unis (ÉAU) par des acteurs de la mondialisation qui tissent un maillage étroit de relations public-privé. Il vise à comprendre dans quelle mesure l'adoption privilégiée d'une nouvelle technique d'agriculture irriguée peut modifier la trajectoire historique d'un système productif agricole et alimentaire.

La notion de trajectoire historique décrit la succession de mutations qu'un système connait au cours de son existence. Les systémistes distinguent deux phénomènes : la bifurcation, qui est la reconfiguration de la structure d'un système en réaction à un changement survenu dans son environnement, et l'émergence, qui désigne la mise en place d'un nouveau jeu d'acteurs, d'une nouvelle structure ${ }^{1}$.

La trajectoire historique du système alimentaire mondial tend, d'après Jean-Louis Rastoin et Gérard Ghersi, vers la coexistence de deux extrêmes : un modèle de plus en plus libéralisé et mondialisé, dominé par un oligopole de firmes transnationales ; et un modèle de proximité, fondé sur des filières courtes, mis en œuvre par des $\mathrm{PME}^{2}$. Le développement des cultures hydroponiques aux ÉAU - pour produire des denrées destinées aux habitants du pays et se substituer autant que faire se peut aux importations - vient brouiller cette analyse puisqu'il coïncide partiellement avec l'essor d'une agriculture de firme : les plantes hydroponiques sont cultivées par des ouvriers agricoles salariés et les exploitations dans lesquelles ils travaillent peuvent être gérées par un consortium.

1. Voir par ex. chez les géographes français : Pumain, 2003, 2017.

2. Rastoin et Ghersi, 2010. 
Notre hypothèse est que la priorité donnée aujourd'hui aux cultures hydroponiques dans le développement agricole aux ÉAU représente une modification sensible de la trajectoire historique du système de production de biens agricoles et alimentaires dans ce pays, une mutation qui est à la fois cause et conséquence de la transformation de la structure sociale de ce système. L'ampleur des capitaux économique et humain nécessaires pour commencer cette technique de culture exclut son adoption dans le cadre d'une agriculture récréative et la réserve à l'agriculture commerciale, ce qui fait apparaître les entrepreneurs comme nouvelle catégorie d'acteurs de la production agricole émiratie. Leur émergence marque un tournant dans ce pays où les cultures n'ont jusqu'ici été qu'une activité de complément et n'ont jamais couvert les besoins des villes côtières, qui ont toujours importé par la mer la majeure partie de leur nourriture ${ }^{3}$. Dans ses aspects techniques, le développement de l'hydroponie vient en outre consommer une rupture déjà largement engagée avec l'ancienne agriculture vivrière oasienne. Les oasis devaient en général leur existence à de longues galeries souterraines creusées en pente douce pour drainer l'eau contenue dans les aquifères. L'eau acheminée jusqu'aux oasis par ces aqueducs souterrains, appelés qanât en Iran et aflâj (au singulier : falaj) aux ÉAU, venait irriguer les parcelles par l'intermédiaire d'un réseau de canaux à l'air libre. Les cultures hydroponiques sont tout à fait différentes puisqu'elles sont irriguées par de l'eau puisée (ou éventuellement de l'eau de mer dessalée) et qu'elles sont hors-sol.

Nous supposons que les entrepreneurs en cultures hydroponiques aux ÉAU s'insèrent dans des réseaux familiaux, tribaux et nationaux dont ils peuvent mobiliser les ressources. Il s'agit donc d'étudier un cas d'embedded entrepreneurship. Cette notion, développée notamment dans la recherche indienne en sciences sociales ${ }^{4}$, rejoint le cadre élaboré par Mark Granovetter qui, à la suite de Karl Polanyi, pose comme principe l'encastrement (embeddedness) des activités économiques dans des réseaux sociaux ${ }^{5}$. Le regard qui en découle sur la figure de l'entrepreneur vient contrebalancer la conception de l'entrepreneur comme acteur autonome capable, grâce à ses caractéristiques individuelles, d'instiguer les transformations de la structure sociale ${ }^{6}$.

3. Voir par ex. Lorimer, 1995, p. 272, ou Cariou, 2017, p. 216.

4. Tripathi, 1971.

5. Granovetter, 1985 par exemple.

6. Voir la présentation de ce débat par exemple chez Giddens, 1979, p. 69-70. 
L'hypothèse sera testée dans un cadre théorique inspiré de la théorie du champ organisationnel formalisée par William Richard Scott qui, dans la voie ouverte par Michel Crozier et Erhard Friedberg ${ }^{7}$, montre comment les acteurs reproduisent $e t$ transforment le contexte dans lequel ils agissent. Le champ organisationnel apparaît comme un intermédiaire entre individus et organisations d'une part et systèmes transnationaux d'autre part. On observe des dynamiques de diffusion et d'imposition dans le sens topdown, d'invention et de négociation dans le sens bottom-up, entre acteurs et champ organisationnel, et entre champ organisationnel et systèmes transnationaux 8 .

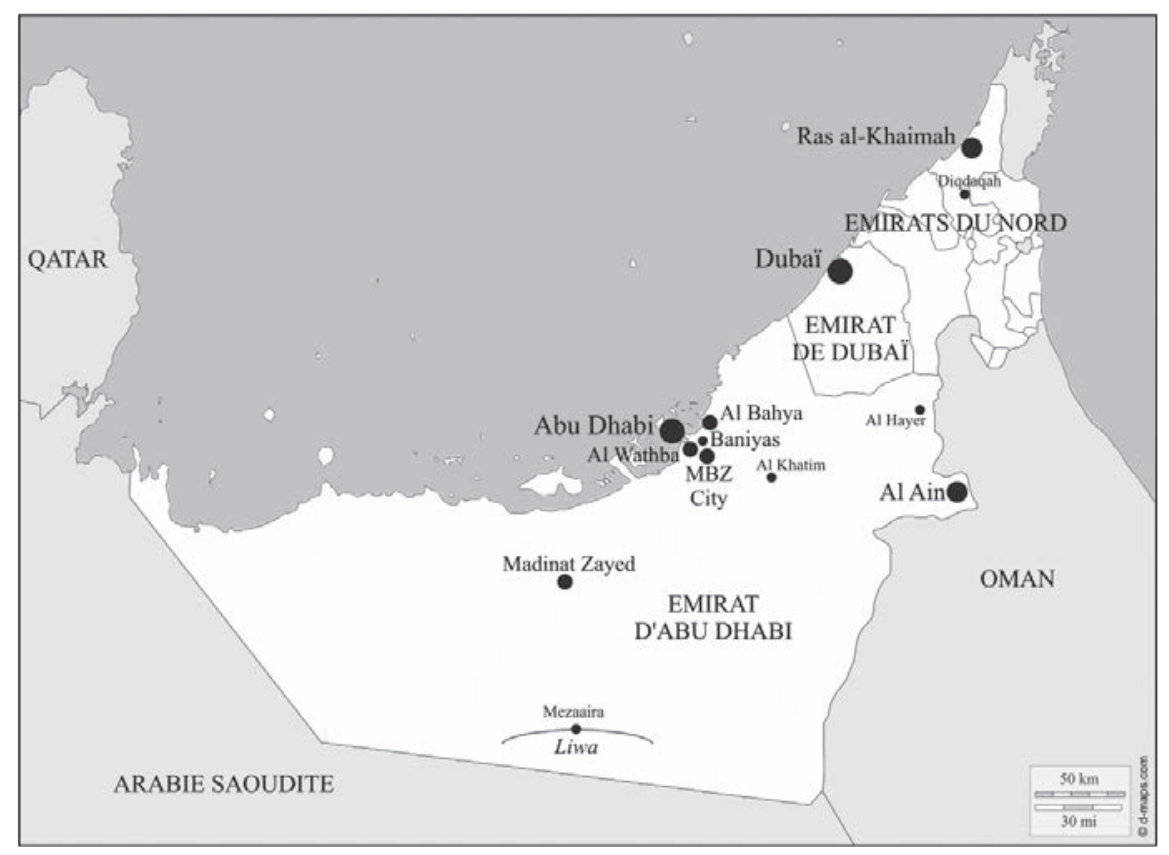

Fig. 1. - Carte des lieux mentionnés dans cet article

Damien Calais

7. Crozier et Friedberg, 1977.

8. Scott, 2008. 
L'article se fonde sur cinq catégories de sources : des données produites par des organisations internationales, des administrations publiques aux ÉAU et des sociétés privées ; des observations effectuées aux ÉAU, au cours de plusieurs séjours en décembre 2015, octobre 2016, octobre 2017, décembre 2017 et février $2019^{9}$; des entretiens avec le propriétaire d'une palmeraie dans le croissant oasien de Liwa (émirat d'Abu Dhabi), cinq ingénieurs agronomes, quatre ouvriers agricoles et trois cadres d'organisations liées au gouvernement d'Abu Dhabi en décembre 2015, septembre-octobre 2016 et février $2019^{10}$; des articles de la presse quotidienne émiratie publiés entre 2012 et 2017 ; des témoignages d'administrateurs et des archives publiques produites à l'époque du protectorat de la GrandeBretagne sur les actuels ÉAU (1892-1971), conservées au centre de Kew, dans le Grand Londres, et consultées en juillet 2016.

\section{La trajectoire historique de l'agriculture moderne aux ÉAU : comprendre la priorité aujourd'hui donnée à l'hydroponie}

\section{Contraintes et potentialités du milieu pour l'agriculture}

Les conditions pédologiques, hydrologiques et climatiques du désert émirati, caractérisé par l'aridité, sont très défavorables aux activités agricoles pour une société qui ne peut artificialiser son milieu. Les sols sont très peu fertiles, peu drainants, balayés par les vents ; les précipitations, très irrégulières, peuvent être plus de 80 fois inférieures à l'évaporation potentielle (à Liwa, par exemple, dans l'émirat d'Abu Dhabi, les précipitations moyennes annuelles sont inférieures à $40 \mathrm{~mm}$ alors que l'évaporation potentielle est proche de $3500 \mathrm{~mm} / \mathrm{an}$ ), ce qui explique en partie la forte minéralisation des eaux souterraines ${ }^{11}$. L'irrigation, obligatoire pour cultiver, doit ainsi

9. Les photographies ont été prises par l'auteur le 30 octobre 2016 dans le secteur d'Al Hayer, émirat d'Abu Dhabi.

10. Nous n’avons hélas pas été en mesure de nous entretenir avec des propriétaires d'exploitations agricoles hydroponiques.

11. Compagnie française des pétroles, 1978 ; Frenken (dir.), 2009, p. 375 ; Cariou, 2017, p. 214. 
suffisamment excéder les besoins des plantes pour lessiver le sol, ce qui aggrave encore la salinisation des nappes ${ }^{12}$.

Sous l'effet d'une forte amplitude thermique journalière, les parasites sont rares et les maladies cryptogamiques presque inexistantes, la fraîcheur nocturne empêchant leur développement. L'ensoleillement permet quant à lui une photosynthèse exceptionnelle. Les rendements du concombre pouvaient ainsi dépasser $60 \mathrm{~kg} / \mathrm{m}^{2}$ dès les années 1970 dans l'émirat d'Abu Dhabi, près du double de ce qui est obtenu aujourd'hui dans les serres françaises les plus performantes ${ }^{13}$. Le désert des ÉAU peut être rendu propice à l'agriculture par des moyens techniques permettant de maîtriser les ressources en eau, au prix toutefois de coûts économiques et environnementaux élevés et d'une dépendance technologique envers l'étranger.

\section{L'introduction de l'agriculture moderne aux ÉAU : un choix politique du gouvernement britannique}

Dans les années 1950, le gouvernement britannique vit s'effriter la légitimité de son protectorat établi en 1892 et qui dura jusqu'à l'indépendance des ÉAU en 1971. C'est dans ce contexte qu'il fut le premier à y investir dans l'agriculture moderne. La sous-alimentation, qui avait été très prononcée dans les villes pendant la Seconde Guerre mondiale, faisait du développement agricole un projet prioritaire ${ }^{14}$. La première étape, en 1955, fut la création d'une station expérimentale et l'ouverture d'une école d'agriculture à Diqdaqah, dans l'émirat de Ras al-Khaimah ${ }^{15}$.

Diqdaqah constitue la mise en œuvre, pour la première fois dans l'agriculture aux Émirats, de l'idéologie du progrès telle qu'elle est née en Europe à l'époque moderne : une volonté d'améliorer la condition humaine grâce au progrès des techniques, fondées sur les sciences plutôt que sur les savoirs pratiques et empiriques des cultivateurs. Sous l'impulsion des responsables de la station, les aflâj, antique système d'irrigation gravitaire,

12. Ghassemi, Jakeman, Nix, 1995.

13. Compagnie française des pétroles, 1978 ; Direction départementale des territoires de Moselle, 2010 .

14. Zacharias, 2017.

15. National Archives (Kew) : FO 371/157041. 
commencèrent à être délaissés au profit de puits munis de pompes motorisées. Les prêts bancaires pour l'achat de celles-ci devaient être garantis par un nantissement, ce qui rendit indispensable l'établissement de titres de propriété ${ }^{16}$. Les cultures auparavant inconnues aux Émirats se multipliaient (tomate, concombre, poivron, etc. ${ }^{17}$. La nouvelle technique d'irrigation et l'importation de semences, principalement des États-Unis ${ }^{18}$, firent entrer l'agriculture des Émirats dans le capitalisme. De bien commun, l'eau souterraine devint un bien ouvert dont le libre accès individualisé échappait au contrôle communautaire. Cette individualisation contrarie l'idée d'un champ organisationnel de l'agriculture moderne aux ÉAU puisque chaque acteur semble jouer son propre jeu sans s'associer aux autres. À notre connaissance, il n'existe d'ailleurs aucune association professionnelle agricole aux ÉAU, qu'il s'agisse de coopératives ou d'associations de défense des intérêts des agriculteurs auprès des autorités.

Dans l'élan du succès de Diqdaqah, le gouvernement de Ras al-Khaimah commença en 1963 à offrir des terres aux citoyens de l'émirat qui en faisaient la demande pour qu'ils y développent une agriculture moderne subventionnée ${ }^{19}$. Nous avons ici un premier exemple d'entrepreneurs agricoles qui, conformément à notre hypothèse, sont insérés dans des réseaux tribaux et nationaux, la reconnaissance du statut de citoyen d'un émirat étant largement liée à l'histoire des allégeances tribales ${ }^{20}$. Cet émirat montagneux semblait propice au développement agricole avec ses piémonts sablo-limoneux et ses précipitations orographiques, mais la teneur en sels minéraux de l'eau issue de l'aquifère atteignait déjà 2000 ppm dans les années 1970 et a subi depuis l'intrusion d'eau marine ${ }^{21}$, ce qui a condamné la majeure partie des exploitations.

16. Zacharias, 2017.

17. Hawley, 1970, p. 239.

18. Zacharias, 2017.

19. El Mallakh, 2015, p. 38.

20. Voir par exemple Heard-Bey, 1999.

21. Frenken, 2009, p. 375. 


\title{
Un développement agricole très subventionné, aujourd'hui remis en question par ses effets environnementaux
}

\begin{abstract}
Abu Dhabi, qui combine de larges ressources en hydrocarbures et un vaste territoire désertique, présente la stratégie agricole la plus ambitieuse des ÉAU. Dans les dix dernières années du règne de Cheikh Zayed (mort en 2004), plus de 20000 parcelles y furent aménagées et distribuées gracieusement par les autorités ${ }^{22}$, qui forèrent les puits pour irriguer les nouveaux périmètres cultivés ${ }^{23}$. Il faut souligner que la plupart des propriétaires des parcelles octroyées n'ont pas choisi l'agriculture comme activité principale ou première source de revenus, et qu'un tiers des parcelles a été abandonné ${ }^{24}$ pour des raisons sur lesquelles nous reviendrons. En dehors des dattes, la production de ces parcelles est rarement commercialisée $e^{25}$. Celles-ci sont donc caractéristiques d'une agriculture récréative et leurs propriétaires sont peu nombreux à pouvoir être qualifiés d'entrepreneurs agricoles.
\end{abstract}

Face aux prétentions saoudiennes sur les oasis d'Al Ain ou de Liwa ${ }^{26}$ et alors que l'agriculture saoudienne était en plein $\operatorname{essor}^{27}$, le développement agricole permettait aux dirigeants émiratis d'affirmer leur pouvoir sur le territoire de leur État. À l'intérieur des ÉAU, le verdissement du désert contribuait à la création d'une identité nationale fondée sur la nostalgie de la vie bédouine $e^{28}$ et qui, par l'évocation des jardins d'al-Andalus ${ }^{29}$, se trouvait reliée à l'identité arabo-musulmane. Il s'agissait aussi de contrôler la croissance urbaine en maintenant tant bien que mal dans leurs villages de sédentarisation les populations bédouines attirées par les métropoles du littoral ${ }^{30}$. La fonction nourricière de l'agriculture était reléguée à

22. Entretien, 26 octobre 2016 ; Cariou, 2017, p. 218-219.

23. Al Abed, Vine, Vine, 1996, p. 131; Al Abed, Vine, 2000, p. 151.

24. Malek, 2013. D'après nos observations, ce chiffre semble plausible.

25. Entretiens, septembre 2016 et 27 octobre 2016. Ceux-ci viennent confirmer des informations plus anciennes: Al Abed, Vine, Vine, 1996, p. 125 ; Al Abed, Vine (dir.), 2000, p. 148.

26. National Archives (Kew): FCO 8/1813; Heard-Bey, 1999, p. 111.

27. Blanc, Brun, 2013, p. 137.

28. Ouis, 2002, p. 45, 293 ; Woertz, 2013, p. 93-98.

29. Voir par exemple Khalid, 2005.

30. Cariou, 2017, p. 218-219. 
l'arrière-plan, la croissance démographique exponentielle rendant chimérique le désir d'autosuffisance alimentaire.

Après avoir été perçue comme une contribution à la sécurité nationale, cette agriculture minière, responsable de $83 \%$ de la consommation d'eau aux ÉAU en $2005^{31}$, apparaît maintenant comme un péril en raison de ses conséquences environnementales ${ }^{32}$. Les pays de la péninsule arabique ont connu une détérioration ${ }^{33}$ et un effondrement de leurs ressources renouvelables en eau souterraine du fait de l'absence ou de l'insuffisance d'encadrement d'une demande en forte croissance ; aux ÉAU, ces ressources par personne et par an ont été divisées par 84 entre 1962 et $2012^{34}$. Les acteurs du secteur de l'agroalimentaire défendent cependant l'attribution de subventions à l'agriculture en jouant sur les angoisses suscitées par la dépendance de plus en plus coûteuse aux importations ${ }^{35}$. La crise alimentaire mondiale de 2007-2008, les contingentements temporaires des exportations de céréales par plusieurs fournisseurs des ÉAU et le risque de perturbation du commerce par un conflit régional ont ravivé les peurs de pénurie alimentaire aux ÉAU.

Afin de répondre à ces inquiétudes sans remettre en cause les privilèges qui soudent une communauté nationale émiratie minoritaire dans son propre pays ${ }^{36}$, l'État apporte des réponses techniques à la dégradation et à la raréfaction de l'eau douce dans les nappes phréatiques. Des nappes fossiles sont désormais exploitées ${ }^{37}$. Le raccordement de parcelles agricoles au réseau de distribution d'eau de mer dessalée est en cours ; les citoyens d'Abu Dhabi et d'autres émirats continuent d'y avoir accès gratuitement alors que la salinisation des eaux du Golfe s'accroît, notamment en raison des rejets de sel par les usines de dessalement ${ }^{38}$. Le développement de l'hydroponie apparaît enfin comme une priorité de l'action gouvernementale. Les ÉAU renouent ainsi avec d'anciennes expériences de culture hors-sol sur leur territoire.

31. Frenken, 2009, p. 376

32. Amery, 2015, p. 18-19.

33. Cariou, 2017, p. 220, p. 223.

34. Amery, 2015, p. 3-4, p. 18-19.

35. Woertz, 2013, p. 13-14.

36. Davidson, 2012 ; Normand, 2011.

37. Al Abed, Vine, Vine, 1996, p. 131.

38. Al Khemeiri, 2018. 


\section{Les cultures hydroponiques, une stratégie pour concilier le développement agricole avec le ménagement des ressources en eau ?}

\section{Saadiyat : la première expérimentation de cultures hydroponiques aux ÉAU}

L'hydroponie a été expérimentée pour la première fois aux ÉAU sur l'île de Saadiyat, à proximité immédiate de la ville d'Abu Dhabi, dans le cadre d'un partenariat entre le gouvernement d'Abu Dhabi et le département des terres arides de l'Université d'Arizona. Il s'agissait de reproduire en l'élargissant une expérience menée à Puerto Peńasco (Mexique). L'institut de recherche, construit entre 1970 et 1972, couvrait deux hectares et comprenait 48 serres en polyéthylène ${ }^{39}$.

On ne recourait pas alors aux hautes technologies comme le contrôle par ordinateur de l'éclairage, de l'apport en solution nutritive ou de la température. Le substrat utilisé était le sable, en raison de son abondance; il a depuis été délaissé au profit du film nutritif ou de la laine de roche, mieux adaptés à la circulation de la solution nutritive en circuit fermé ${ }^{40}$. On effectuait chaque année quatre récoltes de concombres (60\% de la production) et trois de tomates (30\% de la production) ; ces dernières ne poussaient pas en été, faute de climatisation suffisante ${ }^{41}$.

La production était dispendieuse au point que l'on décida d'abandonner une grande partie des cultures hydroponiques pour revenir à des modes de production conventionnels ${ }^{42}$. En 1977, la production des légumes, vendus pour un million et demi de dirhams, avait coûté quatre millions, en raison de l'importance des dépenses en énergie et de la masse salariale (67 salariés, principalement des ouvriers agricoles pakistanais $)^{43}$. L'institut expérimental de Saadiyat fut finalement démantelé, probablement pendant les années 1980.

39. Resh, 2002, p. 253 ; Cordes et Scholz, 1980, p. 17.

40. Resh, 2002.

41. Cordes et Scholz, 1980, p. 18.

42. El Mallakh, 2015, p. 39.

43. Cordes et Scholz, 1980, p. 18-19. 


\section{L'essor récent de l'hydroponie aux ÉAU : un changement technique mais aussi économique et social}

Le développement des cultures hydroponiques est érigé en priorité de la stratégie 2017-2021 pour la sécurité alimentaire élaborée par le ministère du Changement climatique et de l'Environnement des ÉAU ${ }^{44}$ (couramment désigné par son sigle en anglais MOCCAE). Il s'inscrit dans la politique émiratie de développement des hautes technologies, aussi illustrée par l'écoville de Masdar et la centrale solaire Shams. Le discours officiel met en avant la réduction des quantités d'eau nécessaires par rapport aux cultures conventionnelles, de $70 \%$ selon le chiffre le plus communément avancé ${ }^{45}$.

Les engrais, vitaux pour des cultures hors sol, sont fabriqués dans le pays à partir d'hydrocarbures, ce qui questionne la durabilité du développement agricole. Les principales unités de fabrication ont été établies dans les années 1980 et 1990 en joint-venture entre des compagnies émiraties et des firmes transnationales comme Total ou la Sociedad Química y Minera de Chile. Elles réalisent la synthèse de l'ammoniac et de l'urée à partir du gaz exploité par l'Abu Dhabi National Oil Company (ADNOC) dans la Dhafra (région occidentale de l'émirat d'Abu Dhabi) afin de produire des engrais azotés ${ }^{46}$. Les substrats des cultures hydroponiques sont produits en Inde et au Sri Lanka pour la fibre de coco (marque sri-lankaise Riococo) (Fig. 2) et en Europe occidentale pour la laine de roche (fournie par Cultilène, du groupe Saint-Gobain, et par le producteur néerlandais Grodan). Les centrales de ferti-irrigation (Fig. 3) sont aussi de marques néerlandaises (Priva, HortimaX) ${ }^{47}$. Les systèmes de climatisation (cooling pads) viennent d'Europe, des États-Unis, du Canada ou d'Australie. Les quantités d'eau requises pour leur fonctionnement ne sont pas négligeables : dans les conditions estivales habituelles de l'émirat d'Abu Dhabi, il faut $6 \mathrm{~m}^{3}$ d'eau par heure pour climatiser une serre de 32,5 mètres de longueur, de huit mètres de largeur et quatre mètres de hauteur sous chéneaux (dimensions considérées comme optimales pour une température stable

44. Salama, 2017.

45. Al Qaydi, 2016, p. 160-161; Salama, 2017.

46. Al Abed, Vine, Vine, 1996, p. 133 ; ADFERT, 2019; ADNOC, 2019.

47. Entretiens, 30 octobre 2016. 
à l'intérieur de la serre $)^{48}$. La figure 4 rend compte de l'insertion de l'hydroponie émiratie dans la mondialisation par la provenance des différents intrants. Présentée comme la solution à un problème environnemental, le développement de l'hydroponie vient plutôt aggraver les difficultés existantes. Le discours officiel selon lequel l'hydroponie est un instrument de développement durable ne résiste pas à l'épreuve des faits.

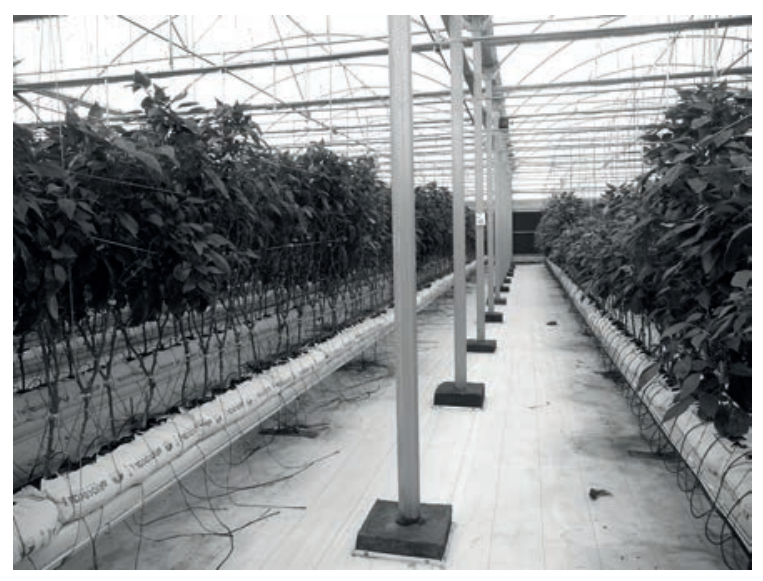

Fig. 2. - La culture hydroponique du poivron sur un substrat de fibre de coco dans une serre à Al Hayer

Cliché Damien Calais

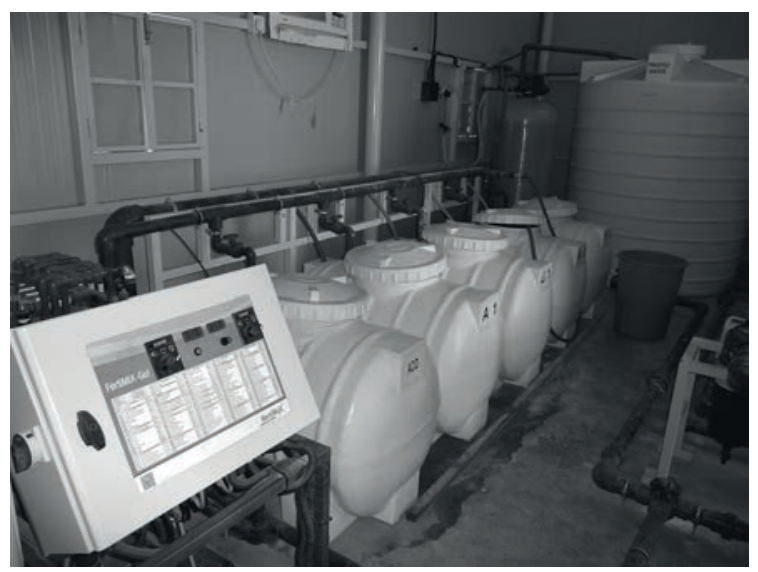

Fig. 3. - La centrale de ferti-irrigation d'une exploitation hydroponique à Al Hayer

Chaque réservoir contient les solutions nutritives requises.

Cliché Damien Calais

48. ADFSC, 2015, p. 64, p. 68. 


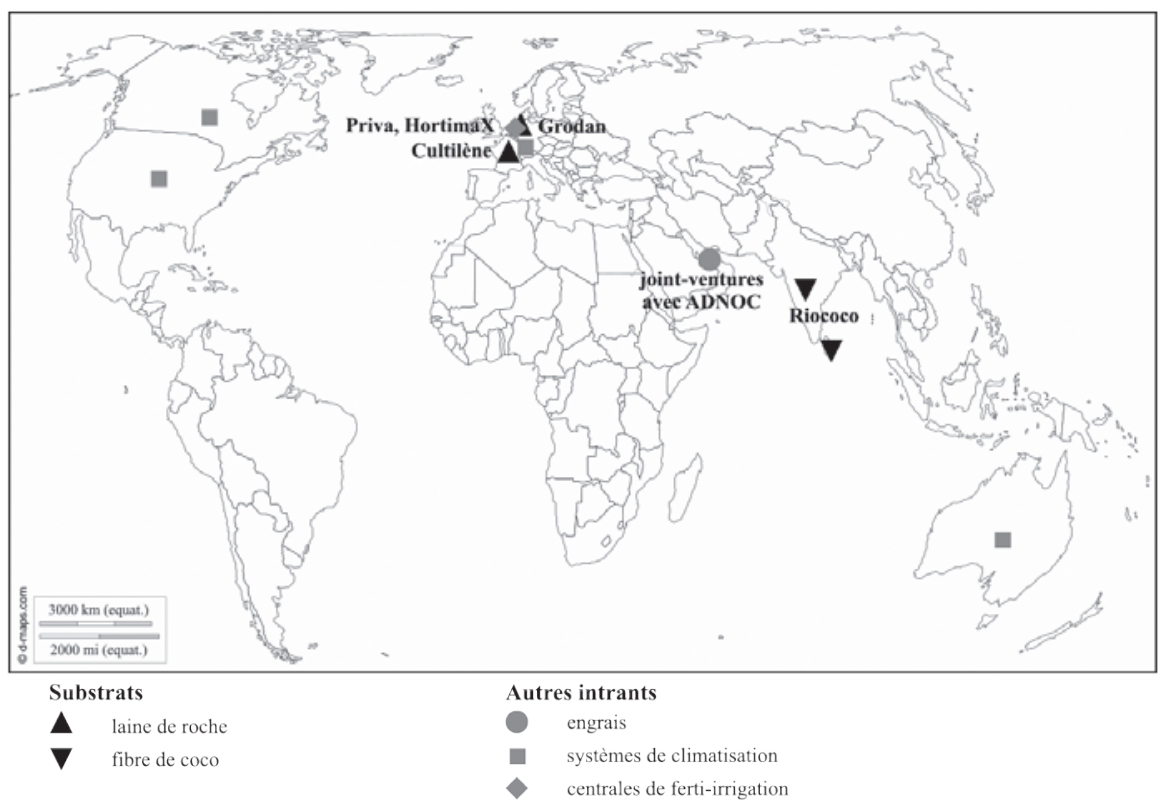

Fig. 4. - Provenance des intrants des cultures hydroponiques émiraties

\section{Damien Calais}

Les stations de recherche gouvernementales conduisent des expériences avec l'aide de partenaires internationaux, souvent là encore néerlandais, afin de réduire les quantités d'eau nécessaires aux cultures hydroponiques ${ }^{49}$. On expérimente à $\mathrm{Al}$ Ain un gel qui se mêle à l'eau afin de limiter l'évaporation $^{50}$. La station de Baniyas développe l'aquaponie, combinaison de pisciculture et d'hydroponie : des tilapias enrichissent par leurs déjections l'eau qui irrigue ensuite les cultures ${ }^{51}$. Des partenaires néerlandais sont également impliqués dans cette quête d'innovations et dans la formation des ingénieurs agronomes ${ }^{52}$, sans lesquels la plupart des exploitations hydroponiques privées aux ÉAU ne sauraient se maintenir.

La première de ces exploitations a été créée en 2005 par des horticulteurs australiens de la société City Farm, renommée en 2008 Emirates Hydroponics Farm (EHF). Les installations, qui couvrent près de

49. Entretiens, 29 décembre 2015 ; Malek, 2014.

50. Entretiens, 29 décembre 2015.

51. Al Qaydi, 2016, p. 160.

52. Malek, 2014. 
deux hectares, se trouvent sur le site d'Al Bahya, proche de la ville d'Abu Dhabi. EHF est spécialisée dans les salades et les herbes aromatiques et fournit des grossistes à Abu Dhabi et Dubaï ainsi que des hypermarchés ${ }^{53}$. D'importants producteurs en hydroponie sont établis dans l'émirat de Ras al-Khaimah, comme Salata, fondé en 2008, dont les fonds propres appartiennent à $80 \%$ à une société d'investissement saoudienne ${ }^{54}$. The Farmhouse s'est établie à Ras al-Khaimah en 2017 pour produire des légumes en hydroponie sans recourir aux pesticides. La production est commercialisée aux ÉAU dans des supermarchés. Le directeur émirati de The Farmhouse est responsable des relations avec les investisseurs du fonds souverain abu-dhabien Mubadala, où a également travaillé le fondateur britannique de la société ${ }^{5}$, ce qui peut laisser supposer que Mubadala est un des financeurs du projet.

L'année 2018 a vu naître un projet d'ampleur inédite conduit à $\mathrm{Al}$ Ain (émirat d'Abu Dhabi) par la branche agricole du Konzern munichois BayWa et le groupe abu-dhabien Al Dahra (qui produit initialement des denrées à l'étranger pour les importer aux ÉAU) : des tomates et d'autres légumes sont cultivés en hydroponie sous deux immenses serres réfrigérées sur un total de dix hectares. La présence à l'inauguration du ministre d'État pour les affaires relatives à la Défense révèle l'importance stratégique de l'hydroponie pour le gouvernement. Destinées au marché émirati, les récoltes s'échelonnent tout au long de l'année et sont commercialisées sous la marque Mahalli ${ }^{56}$. Al Dahra illustre le développement d'une agriculture de firme aux ÉAU. Cette société est une filiale d'Al Ain International Group, holding dont le président, Cheikh Hamdan bin Zayed Al Nahyan, est le demi-frère du souverain d'Abu Dhabi. Cette appartenance tend à valider l'hypothèse de l'insertion des entrepreneurs dans des réseaux familiaux, alors que la présence d'investisseurs étrangers remet celle-ci en question. L'investissement dans l'agriculture sur le territoire émirati est une nouveauté pour Al Dahra, qui possède ou loue des champs, des rizières et des vergers en Europe du Sud et de l'Est, aux États-Unis, au Pakistan

53. Rawlins, 2014.

54. Zeisberger, Prahl, White, 2017, p. 112, p. 118-119.

55. The Farmhouse, 2019.

56. BayWa, 2018 ; Horti Daily, 2018. 
et dans plusieurs pays d'Afrique, et qui possède aussi des parts dans des sociétés de l'industrie agroalimentaire en Inde et en Grèce ${ }^{57}$.

La transformation de la structure du système de production de biens agricoles et alimentaires sur le territoire émirati avec l'entrée de nouveaux acteurs - des investisseurs étrangers, des firmes - entraîne une évolution du rôle attribué à l'agriculture. Il ne s'agit plus pour les ÉAU de s'affirmer par le développement agricole face à l'Arabie saoudite, qui a abandonné sa politique d'autosuffisance ${ }^{58}$ et qui n'exprime plus de velléités sur le territoire émirati. Les hautes technologies utilisées en hydroponie peuvent difficilement susciter la nostalgie de la vie bédouine et les ouvriers agricoles ne sont en aucun cas des Bédouins mais des travailleurs immigrés. L'essor de l'hydroponie oriente l'agriculture émiratie vers la recherche du profit. Il renouvelle par ailleurs la fonction politique de l'agriculture : les héritiers de Cheikh Zayed, célébré comme " père de la nation ", peuvent difficilement pour leur légitimité tourner le dos à la vision de celui qui, connu pour son attachement au développement agricole, est régulièrement cité pour avoir déclaré : "Donnez-moi l'agriculture et je vous donnerai en retour une civilisation ».

L'entrée d'investisseurs étrangers et de firmes transnationales dans la production de cultures hydroponiques aux ÉAU tranche avec le faible attrait des Émiratis pour cette activité, alors que l'agriculture répond à une demande sociale forte. Il nous faut maintenant expliquer les causes de ce contraste.

\section{Le développement de l'hydroponie aux ÉAU confronté à de nombreux défis}

L'hydroponie, une méthode de culture qui requiert d'importants capitaux économiques et humains

Les cultures hydroponiques nécessitent un lourd investissement initial : 75000 dirhams (19000 euros environ) pour une serre de $240 \mathrm{~m}^{2}$, cinq fois

57. Al Dahra, 2019.

58. Blanc, Brun, 2013, p. 137. 
plus qu'avec une serre classique de même surface ${ }^{59}$. Ce coût freine leur adoption. Le secteur d'Al Hayer (émirat d'Abu Dhabi) est représentatif de ce problème : en 2016, sur 409 exploitations agricoles, 75 avaient des serres et deux seulement utilisaient les méthodes hydroponiques ${ }^{60}$. Des mesures gouvernementales ou émanant directement des souverains sont prises pour encourager le développement des cultures hydroponiques, comme le programme "Ziraai " du Khalifa Fund for Enterprise Development, sous la direction du prince héritier d'Abu Dhabi, Cheikh Muhammad bin Zayed Al Nahyan, qui propose depuis 2013 des prêts à taux zéro pour équiper les exploitations de moins de deux hectares ${ }^{61}$.

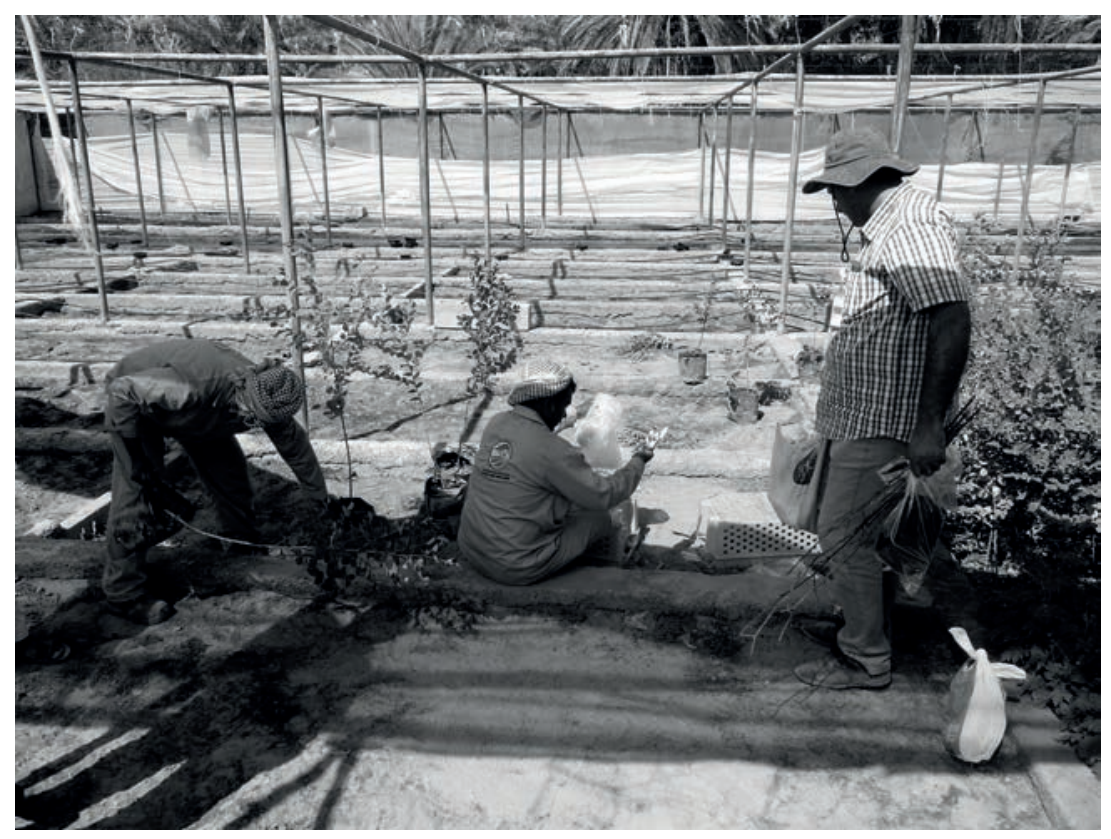

Fig. 5. Un ingénieur agronome égyptien forme des horticulteurs pakistanais au bouturage dans la pépinière du centre de démonstration de l'ADFSC à Al Hayer

Cliché Damien Calais

59. Entretiens, 29 décembre 2015.

60. Entretiens, 30 octobre 2016.

61. Todorova, 2013 ; entretien, 20 février 2019. 
L'hydroponie requiert également un important capital humain. Les ouvriers agricoles sont nombreux dans ce type d'exploitation. Leur encadrement par des ingénieurs agronomes est absolument indispensable au fonctionnement des exploitations hydroponiques. À défaut, les plantes se développent mal et peuvent même mourir très rapidement; de même en cas de panne du système ${ }^{62}$. Les autorités politiques répondent à ce besoin et dispensent les propriétaires des exploitations agricoles d'engager leurs propres ingénieurs, puisqu'ils peuvent recourir aux services d'ingénieurs employés par des organisations gouvernementales, l'Abu Dhabi Farmers' Services Centre (ADFSC) et le MOCCAE, dont les interventions sont gratuites ou facturées à des prix modiques ${ }^{63}$. Ces ingénieurs réalisent aussi des démonstrations comme on le voit sur la figure 5.

Le développement de l'hydroponie n'est par ailleurs possible que là où l'eau puisée contient moins de 2100 ppm de sels minéraux ${ }^{64}$, ce qui devient de moins en moins fréquent aux ÉAU. Les exploitations reliées au réseau d'eau dessalée sont encore rares. Cette contrainte explique la concentration de cultures hydroponiques le long de la route qui relie le croissant oasien de Liwa à la ville de Madinat Zayed, et non dans ce croissant lui-même où l'eau puisée présente une teneur en sels minéraux de 4000 à 14000 ppm pour les deux tiers des puits ${ }^{65}$.

\section{Une agriculture émiratie marginale dans l'économie et la société et qui peut difficilement se substituer aux importations}

Avec la redistribution de la rente pétrolière, les Bédouins qui avaient encore des activités agricoles les abandonnèrent pour trouver en ville un emploi plus rémunérateur, notamment dans le secteur public où les traitements sont sans rapport avec le travail effectué $e^{66}$. Ce délaissement a pu être accéléré par la salinisation de l'eau puisée. Dans l'émirat de Ras al-Khaimah, de nombreux exploitants ont abandonné les cultures destinées à l'alimentation après le doublement des tarifs de l'électricité entre 2010 et 2012 et se

62. ADFSC, 2015, p. 8.

63. Entretiens, 30 octobre 2016.

64. ADFSC, 2015, p. 38.

65. Cariou, 2017, p. 220.

66. Normand, 2011, p. 79. 
sont reconvertis dans la culture des fleurs, dont la valeur ajoutée est beaucoup plus élevée, ou l'élevage, plus rémunérateur ${ }^{67}$. Quant aux citadins qui trouvent dans l'agriculture très subventionnée une source supplémentaire de revenus, ils se contentent d'être propriétaires des parcelles et laissent les travaux agricoles à des salariés immigrés (venus en général d'Asie du Sud) encadrés par des ingénieurs agronomes (souvent originaires d'Égypte ou de Jordanie) employés par des organisations gouvernementales.

Le développement des infrastructures portuaires et routières a par ailleurs facilité l'acheminement de denrées importées, souvent moins chères que celles produites aux ÉAU. D’après nos observations dans les rayons de fruits et légumes de différents points de vente d'Abu Dhabi en octobre 2016, seul le poivron émirati avait un prix inférieur à son équivalent importé. Les exploitations émiraties sont pour la plupart petites (deux à trois hectares), non insérées dans une agriculture sous contrat et insuffisamment équipées pour produire en contre-saison. Celles qui produisent en hydroponie se concentrent sur un petit nombre de cultures à forte valeur ajoutée (laitue, roquette, basilic) ainsi que sur celles qui permettent une rotation rapide et dont les rendements peuvent être augmentés par une culture verticale (tomate, concombre, poivron) ${ }^{68}$ afin d'amortir les coûts de production.

La construction de vastes serres climatisées par de grandes entreprises subventionnées qui peuvent réaliser des économies d'échelle et produire des volumes suffisants pour intéresser la grande distribution pourrait changer la donne. La diversification de la production horticole et sa labellisation sont d'autres stratégies possibles, sur le modèle mis en œuvre dans l'émirat d'Abu Dhabi depuis 2012 : un millier d'exploitations sous contrat avec l'ADFSC vendent leurs produits frais à des centres de collecte suivant des plans de production qui peuvent inclure une quarantaine de fruits et légumes différents ; ces centres contrôlent la qualité des produits et les emballent sous la marque Local Harvest ${ }^{69}$. En 2015, 16 \% des fruits et légumes consommés aux ÉAU étaient produits dans le pays, ce que l'on considérait déjà comme une progression importante ${ }^{70}$. L'autosuffisance paraît cependant hors d'atteinte depuis l'avènement de l'économie pétrolière et du recours massif à

67. Malek, 2012.

68. ADFSC, 2015, p. 6.

69. Entretiens, 26 octobre 2016.

70. Sophia, 2016. 
l'immigration puisque la population a été multipliée par 100 au cours des 60 dernières années et dépasse neuf millions depuis $2013^{71}$.

\section{L'agriculture moderne aux ÉAU : un champ organisationnel en construction et encore discret}

Si l'on considère le système de production de biens agricoles et alimentaires sur le territoire émirati comme un champ organisationnel, tel que l'a formalisé Scott ${ }^{72}$, et qu'on regarde les dynamiques top-down, on constate que celui-ci est soumis par le haut aux forces d'un système transnational, le système alimentaire mondial. Les marchés internationaux, qui fournissent aux ÉAU une large majorité des denrées dont ils ont besoin, imposent à l'agriculture émiratie des contraintes de compétitivité, notamment en matière de coûts de production et de prix à la vente. Le développement récent d'une agriculture de firme aux ÉAU, et notamment de cultures hydroponiques sur de grandes superficies, apparait comme une réponse à cette contrainte. La diffusion des techniques hydroponiques est aussi une dynamique top-down de systèmes transnationaux vers le système productif agricole émirati puisque les technologies utilisées ont été mises au point par des chercheurs occidentaux et que leur mise en œuvre aux ÉAU est assurée par des travailleurs immigrés encadrés par des ingénieurs agronomes eux-mêmes immigrés. Le champ organisationnel de l'agriculture émiratie soumet à son tour les entrepreneurs (qu'ils soient des individus ou de grandes entreprises) à l'impératif environnemental et politique d'économiser la ressource en eau. On a ici une dynamique d'imposition mais aussi de diffusion puisque les ingénieurs qui transmettent les « bonnes pratiques » sont employés par des organisations gouvernementales, après avoir eux-mêmes été formés par des partenaires étrangers.

Si l'on regarde maintenant les dynamiques bottom-up, on voit que les entrepreneurs construisent et diffusent un système d'opinion qui allie l'angoisse de la dépendance aux importations et celle du manque d'eau avec les prétendus atouts environnementaux de l'hydroponie pour justifier le maintien d'une agriculture subventionnée et la priorité donnée à

71. Banque mondiale, 2019.

72. Scott, 2008. 
cette technique de culture. Les entrepreneurs semblent instrumentaliser la figure tutélaire de Cheikh Zayed pour faire du soutien de l'État à l'agriculture un élément de la légitimité des dirigeants. Lorsque les entrepreneurs appartiennent eux-mêmes à une famille régnante, on peut estimer qu'ils jouent la carte politique de l'agriculture en milieu désertique comme expression de puissance et instrument de rayonnement (possiblement en rivalité avec le Qatar qui a annoncé dès 2008 faire de l'essor de l'hydroponie une priorité stratégique ${ }^{73}$ ). Le progrès technique est donc utilisé dans un objectif conservateur. On observe enfin une dynamique d'invention et de négociation du champ organisationnel vers les systèmes transnationaux, puisque gouvernement et entrepreneurs émiratis parviennent à intéresser des partenaires étrangers, parmi lesquels des firmes transnationales, en leur permettant de recevoir des subventions et en leur donnant l'occasion d'expérimenter de nouveaux procédés techniques. On peut aussi supposer que les acteurs émiratis cherchent à ce que leur pays soit identifié sur la scène mondiale comme un pôle d'expérimentation de techniques de pointe, ce qui soutient la diversification de l'économie émiratie.

Y a-t-il bien cependant un champ organisationnel de l'agriculture moderne aux ÉAU ? Les interactions entre acteurs semblent faibles du fait de l'importance de l'agriculture récréative mais l'affirmation d'une agriculture commerciale, notamment à la faveur du développement de l'hydroponie, est de nature à entraîner la construction d'un groupe organisé conscient de ses intérêts et qui se coordonne pour faire valoir ceux-ci. De discrètes mentions dans les publications en témoignent, comme celle d'un membre du Conseil national fédéral (chambre consultative partiellement élue) ayant réclamé un soutien gouvernemental plus franc aux agriculteurs en dehors de l'émirat d'Abu Dhabi qui veulent s'équiper en matériel d'hydroponie ${ }^{74}$, ou celle des concepteurs de l'Abu Dhabi Water Resources Master Plan faisant face au « lobby qui affirme que la poursuite du soutien à l'agriculture [...] est essentielle à la sécurité nationale $»^{75}$.

73. Blanc, Brun, 2013, p. 139.

74. Salama, 2017.

75. EAD, 2009. 


\section{Conclusion}

L'hypothèse formulée en introduction apparaît acceptable mais doit être complétée. Du fait de la dégradation des ressources en eau et du coût élevé de l'investissement initial requis, la réalité de l'adoption des techniques hydroponiques dans l'agriculture émiratie est en deçà de ce que laisse supposer le discours officiel. Il faudra donc plus de recul pour pouvoir dire sur le moyen terme si l'hydroponie aura ou non représenté une mutation suffisamment importante pour modifier la trajectoire historique du système de production de biens agricoles et alimentaires aux ÉAU. Cette possible mutation ne s'inscrira pas dans le long terme puisqu'elle repose sur un mode de développement non durable. Il est cependant certain que le rôle de l'agriculture a été redéfini par l'introduction de cette innovation technique, avec l'affirmation d'une fonction économique d'enrichissement matériel pour les entrepreneurs et le maintien de la fonction politique de l'agriculture.

L'apparition de la figure de l'entrepreneur sur le devant de la scène agricole aux ÉAU est bien à la fois une cause et une conséquence de la priorité donnée à l'hydroponie. Ceux qui se contentent d'une agriculture récréative n'ont pas l'envie de consacrer les moyens nécessaires pour commencer ce type de cultures d'une grande complexité. Celui-ci ne peut être mis en œuvre que par des acteurs véritablement intéressés. Cet intérêt est suscité par l'État à grand renfort de subventions et d'aides diverses. Si les entrepreneurs émiratis sont bien insérés dans des réseaux familiaux, tribaux et nationaux comme le prévoyait notre hypothèse, il faut ajouter que ces entrepreneurs articulent ces réseaux avec ceux de l'économie capitaliste mondialisée. Ces deux catégories de réseaux apportent chacune leurs ressources pour le développement des cultures hydroponiques aux ÉAU et sont complémentaires : les acteurs émiratis de ce développement seraient incapables d'une telle entreprise sans les capitaux économique et humain apportés par leurs partenaires internationaux ; mais ces derniers y consentent parce que les réseaux de ces acteurs émiratis leur permettent de profiter de la redistribution de la rente pétrolière sous forme de subventions accordées à l'agriculture par l'État.

Les entrepreneurs auxquels cet article s'est intéressé apparaissent à la fois comme des objets de la structure sociale, ancrés (embedded) dans leur 
environnement familial et social, et comme des sujets capables de modifier cette même structure pour gagner un nouveau statut qui leur confère des avantages. Modifier la structure, mais sans évidemment la bouleverser, sans quoi ils risqueraient de perdre l'accès à la redistribution de la rente pétrolière. Nous sommes donc en présence d'une bifurcation de la structure sociale plutôt que de l'émergence d'une nouvelle structure. L'embeddedness de ces acteurs économiques est par ailleurs à relativiser puisqu'il n'y aurait rien d'étonnant à les voir abandonner soudainement l'agriculture ou à délocaliser leur activité le jour où les subventions viendraient à s'interrompre, l'Arabie saoudite ayant donné maints exemples de tels revirements.

\section{Sources}

\section{Données produites par des organisations internationales, des administra-} tions publiques aux ÉAU et des sociétés privées :

ADFERT (Abu Dhabi Fertilizer Industries), adfert.com, consulté le 4 juin 2019. ADFSC (Abu Dhabi Farmers' Services Centre), Hydroponics Manual, 2015.

ADNOC (Abu Dhabi National Oil Company), adnoc.ae, consulté le 4 juin 2019. Al ABed Ibrahim, Vine Paula (dir.), United Arab Emirates Yearbook 1999, Trident Press, Londres, 2000.

Al Abed Ibrahim, Vine Peter, Vine Paula (dir.), United Arab Emirates Yearbook 1996, Trident Press, Londres, 1996.

Al DaHra, aldahra.com, consulté en juin 2019.

Ambassade des ÉAU à Paris, Dixième anniversaire de l'État des Émirats arabes unis, 1981.

BANQUe mONDiale, "United Arab Emirates ", data.worldbank.org, consulté le 12 juin 2019.

BAYWA, « Flagship project in the Emirates: BayWa and Al Dahra harvest first premium tomatoes in the desert ", baywa.com, mis en ligne le 24 septembre 2018, consulté le 27 avril 2019.

Compagnie françaIse des pétroles, Agriculture en zone aride. Le Centre expérimental agricole d'Al Ain, Abu Dhabi, Paris, 1978.

EAD (Environment Agency - Abu Dhabi), Abu Dhabi Water Resources Master Plan, Abu Dhabi, 2009.

FRENKEn Karen (dir.), Irrigation in the Middle East Region: AQUASTAT Survey2008, FAO, Rome, 2009.

Horti Daily, "UAE: Al Dahra BayWa greenhouse officialy opened ", hortidaily. com, publié le 29 octobre 2018, consulté le 27 avril 2019.

The Farmhouse, thefarmhouseuae.com, consulté le 27 avril 2019. 


\section{Entretiens menés par l'auteur :}

Entretien, 20 février 2019, avec un responsable du programme "Ziraai » du Khalifa Fund for Enterprise Development, salon Gulfood, World Trade Center de Dubaï.

Entretiens, 30 octobre 2016, avec trois ingénieurs agronomes de l'ADFSC pendant la visite d'exploitations agricoles à Al Hayer (émirat d'Abu Dhabi).

Entretiens, 27 octobre 2016, avec deux ouvriers agricoles à Al Wathba et deux autres ouvriers agricoles à Al Khatim (émirat d'Abu Dhabi).

Entretiens, 26 octobre 2016, avec deux responsables de la communication, un de l'ADFCA et un de l'ADFSC, à Mohammed bin Zayed City (Abu Dhabi).

Entretien, septembre 2016, avec le propriétaire d'une palmeraie à Mezaaira.

Entretiens, 29 décembre 2015, avec deux ingénieurs agronomes de l'ADFSC pendant la présentation d'expérimentations par l'ADFSC au Festival de Wathba (Abu Dhabi).

\section{Articles de la presse quotidienne émiratie :}

KHALID Matein, "History as nostalgia : Spanish Islam and the Arab memory ", khaleejtimes.com, mis en ligne le 8 juin 2005, consulté le 12 juin 2019.

MaLeK Caline, "Experts to tackle challenges of food security in the UAE and Middle East ", thenational.ae, mis en ligne le 28 janvier 2014, consulté le 22 mai 2016.

Malek Caline, "Thousands of abandoned farms across the nation are to be brought back to life ", thenational.ae, mis en ligne le 14 mars 2013, consulté le 12 juin 2019.

MaleK Caline, "Farmers struggle to turn profit as costs bite », thenational.ae, mis en ligne le 3 mars 2012, consulté le 12 juin 2019.

Rawlins Zoe, "Harnessing the wonder of water with hydroponics ", thenational. ae, mis en ligne le 23 octobre 2014, consulté le 12 juin 2019.

Salama Samir, "Hydroponics, organic farming top food security strategy ", gulfnews.com, mis en ligne le 14 mars 2017, consulté le 12 juin 2019.

Sophia Mary, "Sustainable agriculture ", Abu Dhabi Council for Economic Development, adced.ae, mis en ligne en 2016, consulté le 26 juillet 2017.

Todorova Vesela, "Interest free loans for UAE farmers ", thenational.ae, mis en ligne le 4 septembre 2013, consulté le 12 juin 2019.

ZaCHARIAS Anna, "How the Digdagga Experimental Farm in RAK revolutionised regional agriculture in the 1960s", thenational.ae, mis en ligne le 18 août 2017, consulté le 12 juin 2019.

\section{Témoignages d'administrateurs et archives publiques britanniques :}

National Archives (Kew): FCO 8/1813, Claims by Saudi Arabia to territory in United Arab Emirates and Sultanate of Oman, 1972. 
National Archives (Kew): FO 371/157041, Agriculture, 1961, fol. 149-153, 167168 et 192 .

Hawley Donald, The Trucial States, George Allen and Unwin, Londres, 1970.

LORIMER John Gordon, Gazetteer of the Persian Gulf, Oman and Central Arabia.IIGeographical and Statistical, vol. I, Sultan Qaboos University/Garnet, Reading, 1995 (éd. originale : 1908).

\section{Bibliographie}

\section{Ouvrages et articles mobilisés pour le cadre théorique :}

Crozier Michel, Friedberg Erhard, L'Acteur et le système. Les contraintes de l'action collective, Seuil, Paris, 1977.

Giddens Anthony, Central Problem in Social Theory, Macmillan, Londres, 1979.

Granovetter Mark, "Economic Action and Social Structure: The Problem of Embeddedness ", American Journal of Sociology, vol. 91, n 3, 1985, p. 481-510.

Pumain Denise, "De la singularité en géographie », Journal of Interdisciplinary Methodologies and Issues in Science, vol. 1, 2017, DOI : 10.18713/ JIMIS-170117-1-2.

Pumain Denise, "Une approche de la complexité en géographie », Géocarrefour, vol. 78, n 1,2003 , p. 25-31.

Rastoin Jean-Louis, Ghersi Gérard, Le système alimentaire mondial. Concepts et méthodes, analyses et dynamiques, Quae, Versailles, 2010.

Sсотт William Richard, Institutions and Organizations: Ideas and Interests, $3^{\mathrm{e}}$ éd., Sage Publications, Los Angeles, 2008.

Tripathi Dwijendra, "Indian Entrepreneurship in Historical Perspective: A Re-Interpretation ", Economic and Political Weekly, vol. VI, n ${ }^{\circ} 22,1971$, p. 59-66.

\section{Travaux de recherche sur les ÉAU :}

Al KhemeIri Majed, Planification stratégique et développement durable : quel futur pour les Emirats arabes unis ?, thèse de doctorat, Université Paris IV, soutenue le 12 septembre 2018.

Al QAYDi Saif, "The Status and Prospects for Agriculture in the United Arab Emirates (UAE) and their Potential to Contribute to Food Security ", Journal of Basic \& Applied Sciences, vol. XII, 2016, p. 155-163.

Amery Hussein A., Arab Water Security: Threats and Opportunities in the Gulf States, Cambridge University Press, Cambridge (Royaume-Uni), 2015.

Blanc Pierre,Brun Matthieu, «Un regard géopolitique sur l'agriculture de firme dans le monde arabe ", Études rurales, n 191, 2013, p. 129-148.

CAriou Alain, "Liwa: The Mutation of an Agricultural Oasis into a Strategic Reserve Dedicated to a Secure Water Supply for Abu Dhabi », dans Lavie Émilie 
et Marshall Anaïs (dir.), Oases and Globalisation. Ruptures and Continuities, Springer, Cham, 2017, p. 213-225.

Cordes Rainer, Scholz Fred, Bedouins, Wealth, and Change: A Study of Rural Development in the United Arab Emirates and the Sultanate of Oman, The United Nations University, Tokyo, 1980.

DAVIDSON Christopher, "The importance of the unwritten social contract among the Arab monarchies ", New York Times, 29 août 2012.

El Mallakh Ragaei, The Economic Development of the United Arab Emirates, Routledge, Abingdon/New York, 2015 (éd. originale 1981).

Heard-Bey Frauke, Les Émirats arabes unis, trad. Marie-Hélène Colin de Verdière, Karthala, Paris, 1999 (éd. originale : From Trucial States to United Arab Emirates, Longman Group, Londres/New York, 1982).

Normand Alexis, Les Émirats du Golfe, au défi de l'ouverture, L'Harmattan, Paris, 2011.

Ouis Pernilla, Power, Person, and Place: Tradition, Modernity, and Environment in the United Arab Emirates, Lund University in Human Ecology, Lund, 2002. Woertz Eckart, Oil for Food: The global food crisis and the Middle East, Oxford University Press, Oxford, 2013.

\section{Autres ressources consultées :}

202 Direction départementale des territoires de Moselle, «Concombres sous serre ", moselle.gouv.fr, mis en ligne en 2010, consulté le 5 avril 2019.

Ghassemi Fereidoun, Jakeman Anthony John, Nix Henry Allan, Salinisation of Land and Water Resources: Human Causes, Extent, Management and Case Studies, University of New South Wales Press, Sydney, 1995.

Resh Howard M., Hydroponic Food Production, 6e éd., Newconcept Press, Mahwah (New Jersey), 2002.

Zeisberger Claudia, Prahl Michael, White Bowen, Private Equity in Action: Case Studies from Developed and Emerging Markets, Wiley, Chichester, 2017.

\section{L'auteur}

Doctorant en géographie du développement (Université de Paris), Damien Calais prépare une thèse sur la sécurité alimentaire aux Émirats arabes unis sous la direction du Pr. Philippe Cadène. Il est l'auteur de l'article « Abu Dhabi au défi de la sécurité alimentaire. L'approvisionnement des villes comme expression du pouvoir et de la hiérarchie sociale » paru dans le $n^{\circ} 237$ de la Revue internationale des études du développement (premier trimestre 2019). Il est professeur d'histoire-géographie au lycée Jean-Monnet de Crépy-en-Valois (Oise). Contact : damien.calais@live.fr 